\title{
Novel interactions of TG2 with heparan sulfate proteoglycans: reflection on physiological implications
}

\author{
E.A.M. Verderio ${ }^{1 *}$, A. Scarpellini ${ }^{1}$ and T. Johnson ${ }^{2}$ \\ ${ }^{1}$ School of Science and Technology, Nottingham Trent University, Clifton Lane, Nottingham, \\ United Kingdom. \\ ${ }^{2}$ Academic Nephrology Unit (Sheffield Kidney Institute), The University of Sheffield \\ Beech Hill Road, Sheffield, United Kingdom. \\ *Author for correspondence (e-Mail: elisabetta.verderio-edwards@ntu.ac.uk).
}

\section{Keywords}

Transglutaminase-2; Heparan sulphate proteoglycans; syndecan-4; wound healing; fibrosis. 


\begin{abstract}
This mini-review brings together information from publications and recent conference proceedings that have shed light on the biological interaction between transglutaminase-2 and heparan sulphate proteoglycans. We subsequently draw hypothesis of possible implications in the wound healing process. There is a substantial overlap in the action of transglutaminase- 2 and the heparan sulphate proteoglycan syndecan-4 in normal and abnormal wound repair. Our latest findings have identified syndecan-4 as a possible binding and signalling partner of fibronectinbound TG2 and support the idea that transglutaminase- 2 and syndecan- 4 acts in synergy.
\end{abstract}


Role of transglutaminase-2 in wound healing: open questions on its externalisation. Transglutaminases catalyse the post-translation modification of proteins predominantly via crosslinking glutamine and lysine amino acids on adjacent polypeptides (Lorand and Graham 2003). These covalent $\varepsilon$ - $(\gamma$-glutamyl) lysine crosslinks are stable and resistant to enzymatic, chemical and mechanical disruption (Lorand and Conrad 1984). Several transglutaminases have been characterised with distinct genes, structures and physiological functions (Lorand and Graham 2003). Examples include the plasma factor XIIIa involved in crosslinking fibrin during clot formation and the keratinocyte transglutaminase involved in the formation of the cornified envelope during terminal differentiation. The tissue transglutaminase or transglutaminase-2 (TG2), the most widespread member of this family, is present in many different cell types and has been implicated into a plethora of biological functions (Fesus and Piacentini 2002; Griffin et al. 2002; Lorand and Graham 2003), among which the role of TG2 in the normal and abnormal wound healing response is well established in vitro and shown in vivo (Verderio et al. 2005). The predominant role of TG2 in a wound response seems to be in stabilising the cells' extracellular environment, by affecting the extracellular matrix (ECM) composition and altering the cells' adhesive and migration potential. The chronic over expression and cellular secretion of TG2 has been observed in numerous scarring and fibrotic-like disease states which are the result of continuous insult and chronic repair. These include kidney fibrosis (Johnson et al. 2003; Johnson et al. 1997; Johnson et al. 1999), liver fibrosis (Grenard et al. 2001; Qiu et al. 2007), pulmonary fibrosis (Griffin et al. 1979; Richards et al. 1991), atherosclerosis (Sane et al. 2007), myocardial myopathy (Small et al. 1999) and nephrogenic systemic fibrosis (Parsons et al. 2007). In experimental 
renal scarring, the predominant role of TG2, once secreted by tubular epithelial cells or mesangial cells, is associated with accumulation of ECM, both indirectly via TG2mediated fibroblast adhesion and migration into the damaged area, and directly by the formation of $\varepsilon$ ( $\gamma$-glutamyl) lysine dipeptide bonds within the ECM (Verderio et al. 2005). TG2 crosslinking subsequently accelerates the rate of collagen deposition by non conventional pathways, while conferring the ECM with resistance to matrix metalloproteinase degradation (Fisher et al. 2005).

In this process, it remains unclear how TG2 is exported from the cell and released into the extracellular environment and targeted to the cell surface and ECM, since TG2 does not have a signal peptide and thus ER/Golgi dependent N-glycosylation can not occur (Gentile et al. 1991; Lorand and Graham 2003). TG2 must therefore be trafficked by a non-classical secretion route. Although a key trigger for TG2 export is cell stress, TG2 is not released non-specifically due to cell death/damage, since extracellular trafficking occurs in the absence of leakage of intracellular components and cells remain viable ( $\mathrm{Li}$ et al. 2002; Skill et al. 2004). Once exported into the ECM, TG2 appears to have an additional non enzymatic structural adhesive role in reinforcing the integrin-fibronectin $(\mathrm{FN})$ interaction in a way which is independent from the ArgGlyAsp "RGD" cell binding domain of fibronectin (Verderio et al. 2003). However, while we know that TG2 can associate with integrin $\beta 1$ and $\mathrm{FN}$ at the cell surface (Akimov et al. 2000), exactly how TG2 at these sites is controlled is unknown. We initially reported that TG2-mediated RGD-independent cell adhesion is based on its interaction with cell-surface heparan sulphate (HS) chains (Verderio et al. 2003). In the course of the past few years, new observations have accumulated which 
support the hypothesis that the biological role of TG2 may be modulated by its interaction with HS proteoglycans (HSPG)s.

\section{Possible role of heparan sulfates in mediating the biological action of TG2.}

HSPGs are specialised glycoproteins with a core protein linked to one or more HS glycosoaminoglycans (GAG) chains, which are linear polysaccharides consisting of alternating $\mathrm{N}$-acetylated or $\mathrm{N}$-sulphated glucosamine units, and uronic acids (Bishop et al. 2007). The assembly of HS chains on core proteins, which is catalysed by enzymes of the Golgi, leads to a wide structural heterogenicity (e.g. chain size and length, extent of sulphation, spatial distribution of negatively charged groups etc.). Bergamini and collegues have shown that TG2 has some affinity for the heparansulfate analogue heparin, since a heparin-Sepharose column was used in the last step of purification of TG2 from erythrocytes (Signorini et al. 1988). They subsequently showed that binding of TG2 to heparin does not affect the catalytic activity of the enzyme but protect it from thermal unfolding and proteolytic degradation (Gambetti et al. 2005). Interestingly, adhesive ECM glycoproteins such as FN and vitronectin, and growth factors such as midkine, which all serve as substrates for TG2 transamidation, are also heparin-binding proteins. In some cases, the TG2-mediated protein transamidation can be even augmented by GAGs such as heparin (Sane et al. 1990), suggesting that heparin could help immobilise TG2 on these target substrates.

In collaboration with Dr Lortat-Jacob (Institut de Biologie Structurale CNRS-CEA, Grenoble), we have recently studied the interaction of TG2 for HS using, for the first time, a physiologically relevant assay based on surface plasmon resonance (SPR). This assay demonstrated high affinity of recombinant human TG2 for heparin 
(Scarpellini et al. 2007), suggesting the presence of heparin binding sites within TG2. The negative charge of HS chains makes them associate with high affinity to basic amino acids. Work by Cardin and Weintraub (Cardin and Weintraub 1989) has led to the identification of heparin-binding consensus sequences, amongst which the putative heparin-binding motif $\mathrm{XBBXB}$ ( $\mathrm{B}$ is a basic amino acids and $\mathrm{X}$ a hydropathic amino acid) can be found in human TG2 (261-65). This TG2 site appears to be accessible to HS chains, moreover it is conserved among different species (human, rat, mouse, guinea pig) (Figure 1). However, it seems s to be specific for TG2 since it is not conserved in human Tranglutaminase-1, -5, -7 and Factor XIIIa; the motif is also not conserved in Transglutaminase- 3 and -6 , however in this case it is replaced by basic amino acids which may have some affinity for HS (data not shown). Apart from the $\mathrm{XBBXB}$ motif described above, there are other candidate clusters rich in Lys and Arg which might function as possible HS binding sites, although the TG2 protein is relatively acidic overall, and based on the electrostatic surface there are no very obvious domains.

Experiments with cells in culture have shown that heparitinase digestion of cellsurface HS, but not digestion of choindrotin sulphates, impairs the RGD-independent cell spreading and actin assembly mediated by FN-bound TG2 (Verderio et al. 2003). However, digestion of HS did not significantly affect actin assembly mediated by FN (Verderio et al. 2008), consistent with previous reports showing that HS-deficient syndecan-4 core proteins are sufficient to support the formation of stress fibers (Echtermeyer et al. 1999). Hence, our findings suggest that cell-surface HS are essential to support TG2-mediated RGD-independent cell adhesion. 
In summary, the affinity of TG2 for HS as demonstrated by (i) plasmon surface resonance, (ii) the presence of at least one heparin binding motif in TG2 and (iii) the loss of TG2-mediated RGD-independent adhesion following HS digestion, gives strong support to the hypothesis of TG2 binding to cell-surface HSPGs receptors.

Syndecan-4 is a candidate co-receptor for TG2. There are three main subclasses of HSPGs, which may all potentially interact with TG2 (Bishop et al. 2007). The HSPGs belonging to the Syndecan family of cell-surface receptors consist of a core protein embedded in the membrane lipid bilayer and glycoproteins extending into the extracellular environment. Syndecans typically function as receptors for signalling peptides such as fibroblasts growth factor (FGF), vascular endothelial growth factor (VEGF) and transforming growth factor- $\beta$ (TGF $\beta$ ). Glypicans are HSPGs attached to the cell surface via a glycophosphatidyl-inositol (GPI) membrane anchor, and have a critical function in developmental morphogenesis (Filmus and Selleck 2001). Perlecans are among a class of secreted proteoglycans found in the basement membranes and are predominantly associated with generating a negatively charged membrane to modify membrane transport e.g. in the glomerular basement membrane (Bishop et al. 2007).

Transgenic mice with a defect in the expression of the Syndecan family member Syndecan-4 and Syndecan-1 are healthy, fertile but present delayed wound healing (Echtermeyer et al. 2001; Stepp et al. 2002). Syndecan-1 is principally involved into keratinocyte migration and re-epithelization, while syndecan-4 is important in fibroblasts migration. The viable phenotype may be due to the variety of HS present in cells which may compensate for the lack of Syndecan-1 or -4 at the cell surface. In 
fact, targeted deletion in mice of $\mathrm{N}$-acetylglucosamine $\mathrm{N}$-deacetylase-Nsulfotransferase (Ndst), the enzyme which catalyzes the first sulfation step in the synthesis of HS, has resulted into embryonic lethality (Fan et al. 2000). Among all the proposed functions, a key role of cell-surface HSPGs is that of regulating the activity of fibroblast growth factor 2 (FGF2), by facilitating the interaction of FGF-2 with its receptor through a low but important affinity with HS. This finding is supported by crystallographic structures of heparan sulfate associated with an FGF in complex with its receptor (Pellegrini et al. 2000).

Among the subfamilies of HSPGs, a possible candidate receptor for TG2 in cell adhesion and migration is Syndecan 4 (S4), which is the only member of the Syndecan family to be consistently present in focal adhesions (Morgan et al. 2007). S4 acts as a co-receptor for both ECM components and soluble ligands (growth factors). In response to an ECM ligand, the S4 cytoplasmic domain binds phosphatidylinositol 4,5, biphosphate and directly activates protein kinase $\mathrm{C} \alpha$ (PKC $\alpha$ ) to promote cellular spreading (Oh et al. 1997). Therefore, the binding of TG2 to S4 is consistent with the requirement of $\mathrm{PKC} \alpha$ activity to support TG2mediated RGD-independent cell adhesion, demonstrated by the finding that a PKC inhibitor with high specificity for PKC $\alpha$ can block this adhesive pathway (Verderio et al. 2003). However, the signalling activity of S4 at focal adhesions is not exclusive for PKC $\alpha$. It also leads to the activation of focal adhesion kinase (FAK), as demonstrated by the lower phosphorylation levels of FAK $\operatorname{Tyr}(397)$ in fibroblasts derived from syndecan-4-null mice allowed to adhere on FN for 3 hours, and by the rescuing of FAK Tyr(397) phosphorylation by re-expression of syndecan-4 (Wilcox-Adelman et al. 2002). We have now confirmed that syndecan-4-null fibroblasts have a lower level 
of FAK Tyr(397) also in the starting phase of cell adhesion to FN (40-minutes adhesion), compared to wild-type cells (Verderio et al. 2008). In this initial phase of adhesion, FAK is responsible for the early downregulation of the small GTPase RhoA, event which facilitates cell spreading and migration (Ren et al. 1999). Interestingly, we measured an analogue defect in early FAK $\operatorname{Tyr}(397)$ phosphorylation in TG2-null mouse embryonic fibroblasts, compared to wild type fibroblasts, which was accompanied by hyperactivation of RhoA and decreased cell migration (Verderio et al. 2008) . This similarity between the interference of S4 and TG2 function in early FAK activation, could be explained by the cooperation between TG2 and S4 at focal adhesion. Indeed, recent work in our laboratories has demonstrated the association of S4 and TG2 in cell membranes of human osteoblasts (HOB), a cell type known to be rich in RGD-independent, HS-mediated adhesive pathways (Verderio et al. 2003), which could explain their convergence of action. In this study, we have shown that TG2 is present in S4 immunoprecipitates of wild type and TG2-transfected human osteoblasts (HOB-TG14) (Verderio et al. 2001) and that pre-treatment of cells with heparitinase lowers the amount of co-immunoprecipitated TG2. This however, is not decreased by pre-incubation of cells with competitive concentrations of a peptide that mimics the FN binding site within TG2 (Hang et al. 2005), and thus inhibits TG2-FN binding (Scarpellini et al. 2007), suggesting a direct interaction of TG2 with S4. We have also began demonstrating the S4-TG2 association in cell membranes of stably transformed Swiss 3T3 cell lines, generated by transfecting TG2 cDNA under the control of a tetracycline-regulated inducible promoter (unpublished data). These findings collectively indicate that TG2 associates with the HS chains of S4 and that this interaction is direct and not mediated by heparin-binding FN. Furthermore, we have found that S4 is required to mediate the 
adhesive action of matrix TG2, since S4-null dermal fibroblasts, originating from S4 knockout mouse (Ishiguro et al. 2000), display a significantly lower level of RGDindependent focal adhesion assembly and phosphorylation of FAK at Tyr397 compared to wild type fibroblasts (Verderio et al. 2008). Work from Griffin and coworkers have shown that not only S4, but also Syndecan-2 is a potential cell-surface receptor for TG2 in HOB (personal communication).

Collectively, these findings demonstrate the direct association of TG2 with S4 and the dependence of TG2 on S4 in cell adhesion, supporting the hypothesis that S4 may function as a co-receptor for TG2.

\section{Do TG2 and Syndecan 4 interact in vivo?}

It is apparent that both TG2 and S4 are implicated in similar functions in cell-ECM interactions that are important for wound repair. In injured mice (skin wounding), S4 is overexpressed in many cell types in the granulation tissue (Gallo et al. 1996). The activity of TG2 is also found to be significantly increased at day 1-3 post skin wounding in rat (Haroon et al. 1999). A role of S4 in wound healing is supported by the finding of delayed wound repair in S4-null mice, as previously discussed (Echtermeyer et al. 2001). Similarly, delayed wound healing was reported in TG2-null mice by R Graham and colleagues (Mearns et al. 2002). These defects in repair associated with $\mathrm{S} 4$ and TG2 deletion can be reproduced in vitro utilising a "wound scratch assay" with either S4-null fibroblasts originating from S4 knockout mouse (Echtermeyer et al. 2001) or TG2-null fibroblasts generated by antisense RNA (Stephens et al. 2004) or derived from TG2-knockout mouse (Verderio et al. 2008). TG2 and S4 both appear to be implicated in conditions of continuous insult leading to 
tissue fibrosis e.g. kidney scarring. Chronic over-expression and cellular secretion of TG2 is a major contributing factor to the pathogenesis of kidney scarring (Johnson et al. 2007). An increase in total HSPGs was also found in the glomerulus and tubulo interstitium of renal biopsies of patients with renal disease (Morita et al. 1994). More specifically, the HSPG member S4 (Lorand and Conrad 1984) was found to be upregulated in IgA nephropathy (Yung et al. 2001) and diabetic nephropathy (Fan et al. 2003). These functional similarities in vivo are consistent with the interaction of TG2 and S4 that we have begun to demonstrate in vitro, and could possibly be explained by a synergistic action. However, to demonstrate whether TG2 and S4 interact in vivo, it is important to determine the dependence on each other for these roles. Our preliminary studies have shown a reduced level of fibrosis (quantified by Masson's trichrome staining) in sections of kidney from S4-knockout mice subjected to experimental scarring induced by unilateral ureteric obstruction (UUO), compared to similarly treated wild type inbred C57BL/6J mice. The protection of kidney fibrosis caused by S4 deletion is consistent with its over-expression in fibrotic tissue (Morita et al. 1994), and is reminiscent to that seen in TG2 knockout mice (Mohammed et al. 2006). Our further observation, that $\mathrm{S} 4$ deletion is associated with a significant reduction in extracellular TG activity in kidney sections of S4 knockout mice compared to WT mice post UUO, suggests that S4 and TG2 may cooperate in the repair process (Scarpellini, Johnson\&Verderio, unpublished data).

\section{Perspectives}

A substantial body of new observations has confirmed the affinity of TG2 for HS, which is a common component of the ECM (Bishop et al. 2007), and has begun to define the physiological significance of the affinity of TG2 for HS chains. As 
discussed, we have identified the HS proteoglycan S4 as one possible co-receptor of TG2 on the cell surface of fibroblasts and osteoblasts (Scarpellini et al. 2008; Verderio et al. 2008). This finding forms the hypothesis of a possible cooperative action between TG2 and S4 in vivo, in normal and excessive repair processes. Given the known affinity of TG2 for integrin receptors (Akimov et al. 2000), and their synergistic action with $\mathrm{S} 4$, one avenue of research would be that of identifying the signalling pathways induced by TG2 engagement to both receptors. Furthermore, the interaction of TG2 with the other members of HSPGs subfamilies remains to be investigated. Indeed, the wide range of HSPGs raises the possibility of different TG2HS proteoglycan combinations depending on cellular context. Furthermore, the great structural heterogeneicity of the HS chains with possible specialised function (Seeberger and Werz 2007) generates the question of whether TG2 binds selectively to a preferential structure. Another challenge in the field would be that of defining the mechanism of functional cooperation between S4 and TG2. Examples of mechanisms could include a possible role of HS in the localisation and cell surface trafficking of TG2. Interestingly, one well-known binding partner of HS is the leaderless secreted protein FGF-2. A recent report by Zehe et al (Zehe et al. 2006), has shown that cellsurface HS are necessary to guarantee FGF-2 secretion and that they would probably contribute to secure FGF-2 translocation through the plasma membrane by acting as "molecular traps". In the light of these findings, it would be useful to establish the role of HSPGs in TG2 localisation and cell trafficking as they may be involved in the unconventional secretion process of TG2 through a yet to be discovered mechanism. Indeed, given the length and flexibility of the HS chain, e.g. HSPGs can bind distant ECM components and even act across cells by binding and localising ligands on adjacent cells (Bishop et al. 2007), the HS chains could contribute to increase the 
local concentration of TG2 and consequently its biological activity, as hypothesised in Figure 2. The association of TG2 with integrin receptors at the cell surface was initially described by Belkin and co workers (Akimov et al. 2000). We have now shown its further association with $\mathrm{S} 4$, raising the possibility of TG2 contributing to cell adhesion, via binding to the S4 ectodomain. Telci et al. (Telci et al. 2007) have recently presented that RGD-independent cell adhesion mediated by FN-bound TG2 depends on integrin $\beta 1$, as the process is impaired in $\beta 1$-null fibroblasts and it is restored by $\beta 1$ re-expression. The concomitant interaction of TG2 with S4 and integrin receptors may have implications for their cooperative signalling, that could be dependent on the receptors' spatial localisation.

In conclusion, increased knowledge of TG2-HSPG interaction may contribute to answer open questions on TG2 localisation and trafficking and help design novel strategies for the specific inhibition of TG2 action, with important applications in early intervention against tissue scarring and fibrosis. 


\section{Acknowledgements}

We wish to thank Dr H. Lortat-Jacob and Dr C. Laguri (Institut de Biologie Structurale CNRS-CEA, Grenoble) for useful comments on the TG2 structure and potential heparin binding sites. 


\section{References}

Akimov SS, Krylov D, Fleischman LF, Belkin AM (2000) Tissue transglutaminase is an integrin-binding adhesion coreceptor for fibronectin. J Cell Biol 148:825-38.

Bishop JR, Schuksz M, Esko JD (2007) Heparan sulphate proteoglycans fine-tune mammalian physiology. Nature 446:1030-7

Cardin AD, Weintraub HJ (1989) Molecular modeling of protein-glycosaminoglycan interactions. Arteriosclerosis 9:21-32

Echtermeyer F, Baciu PC, Saoncella S, Ge Y, Goetinck PF (1999) Syndecan-4 core protein is sufficient for the assembly of focal adhesions and actin stress fibers. J Cell Sci 112 ( Pt 20):3433-41

Echtermeyer F, Streit M, Wilcox-Adelman S, Saoncella S, Denhez F, Detmar M, Goetinck P (2001) Delayed wound repair and impaired angiogenesis in mice lacking syndecan-4. J Clin Invest 107:R9-R14

Fan G, Xiao L, Cheng L, Wang X, Sun B, Hu G (2000) Targeted disruption of NDST1 gene leads to pulmonary hypoplasia and neonatal respiratory distress in mice. FEBS Lett 467:7-11

Fan Q, Shike T, Shigihara T, Tanimoto M, Gohda T, Makita Y, Wang LN, Horikoshi S, Tomino Y (2003) Gene expression profile in diabetic KK/Ta mice. Kidney Int 64:1978-85

Fesus L, Piacentini M (2002) Transglutaminase 2: an enigmatic enzyme with diverse functions. Trends Biochem Sci 27:534-9

Filmus J, Selleck SB (2001) Glypicans: proteoglycans with a surprise. J Clin Invest 108:497-501

Fisher M, Huang L, Hau Z, Griffin M, El Nahas AM, Johnson TS (2005) Over Expression of Tissue Transglutaminase in Proximal Tubular Epithelial Cells Affects ECM accummulation in vitro. Proceedings of the 3rd World Congress in Nephrology, Singapore:W-P020031

Gallo R, Kim C, Kokenyesi R, Adzick NS, Bernfield M (1996) Syndecans-1 and -4 are induced during wound repair of neonatal but not fetal skin. J Invest Dermatol 107:676-83

Gambetti S, Dondi A, Cervellati C, Squerzanti M, Pansini FS, Bergamini CM (2005) Interaction with heparin protects tissue transglutaminase against inactivation by heating and by proteolysis. Biochimie 87:551-5

Gentile V, Saydak M, Chiocca EA, Akande O, Birckbichler PJ, Lee KN, Stein JP, Davies PJ (1991) Isolation and characterization of cDNA clones to mouse 
macrophage and human endothelial cell tissue transglutaminases. J Biol Chem 266:478-83

Grenard P, Bresson-Hadni S, El Alaoui S, Chevallier M, Vuitton DA, Ricard-Blum S (2001) Transglutaminase-mediated cross-linking is involved in the stabilization of extracellular matrix in human liver fibrosis. J Hepatol 35:367-75

Griffin M, Casadio R, Bergamini CM (2002) Transglutaminases: nature's biological glues. Biochem J 368:377-96

Griffin M, Smith LL, Wynne J (1979) Changes in transglutaminase activity in an experimental model of pulmonary fibrosis induced by paraquat. $\mathrm{Br} \mathrm{J}$ Exp Pathol 60:653-61

Haroon ZA, Hettasch JM, Lai TS, Dewhirst MW, Greenberg CS (1999) Tissue transglutaminase is expressed, active, and directly involved in rat dermal wound healing and angiogenesis. Faseb J 13:1787-95

Johnson TS, El-Koraie AF, Skill NJ, Baddour NM, El Nahas AM, Njloma M, Adam AG, Griffin M (2003) Tissue transglutaminase and the progression of human renal scarring. J Am Soc Nephrol 14:2052-62

Johnson TS, Fisher M, Haylor JL, Hau Z, Skill NJ, Jones R, Saint R, Coutts I, Vickers ME, El Nahas AM, Griffin M (2007) Transglutaminase inhibition reduces fibrosis and preserves function in experimental chronic kidney disease. J Am Soc Nephrol $18: 3078-88$

Johnson TS, Griffin M, Thomas GL, Skill J, Cox A, Yang B, Nicholas B, Birckbichler PJ, Muchaneta-Kubara C, Meguid El Nahas A (1997) The role of transglutaminase in the rat subtotal nephrectomy model of renal fibrosis. J Clin Invest 99:2950-60.

Johnson TS, Skill NJ, El Nahas AM, Oldroyd SD, Thomas GL, Douthwaite JA, Haylor JL, Griffin M (1999) Transglutaminase transcription and antigen translocation in experimental renal scarring. J Am Soc Nephrol 10:2146-57.

Li X, Verderio E, Griffin M (2002) Effects of tissue transglutaminase expression on cell stress induced by 3-nitropropionic acid (3NP). Minerva Biotecnologica 14:209

Lorand L, Conrad SM (1984) Transglutaminases. Mol Cell Biochem 58:9-35.

Lorand L, Graham RM (2003) Transglutaminases: crosslinking enzymes with pleiotropic functions. Nat Rev Mol Cell Biol 4:140-56

Mearns B, Nanda N, Michalicek J, Iismaa S, Graham R (2002) Impaired wound healing and altered fibroblast cytoskeletal dynamics in Gh knockout mice. Minerva Biotecnologica 14:218

Mohammed N, Haylor J, Hau Z, El Nahas A, Griffin M, Johnson T (2006) Knockout of Transglutaminase Type 2 Slows the Development of Kidney Scarring in the Mouse 
UUO Model. Proceedings of the 2006 Renal Association Annual Conference, May, Harrogate, UK www.renal.org:RA6066

Morgan MR, Humphries MJ, Bass MD (2007) Synergistic control of cell adhesion by integrins and syndecans. Nat Rev Mol Cell Biol 8:957-69

Morita H, David G, Mizutani A, Shinzato T, Habuchi H, Maeda K, Kimata K (1994) Heparan sulfate proteoglycans in the human sclerosing and scarring kidney. Changes in heparan sulfate moiety. Contrib Nephrol 107:174-9

Oh ES, Woods A, Couchman JR (1997) Syndecan-4 proteoglycan regulates the distribution and activity of protein kinase C. J Biol Chem 272:8133-6

Parsons AC, Yosipovitch G, Sheehan DJ, Sangueza OP, Greenberg CS, Sane DC (2007) Transglutaminases: the missing link in nephrogenic systemic fibrosis. Am J Dermatopathol 29:433-6

Pellegrini L, Burke DF, von Delft F, Mulloy B, Blundell TL (2000) Crystal structure of fibroblast growth factor receptor ectodomain bound to ligand and heparin. Nature 407:1029-34

Qiu JF, Zhang ZQ, Chen W, Wu ZY (2007) Cystamine ameliorates liver fibrosis induced by carbon tetrachloride via inhibition of tissue transglutaminase. World $\mathrm{J}$ Gastroenterol 13:4328-32

Ren XD, Kiosses WB, Schwartz MA (1999) Regulation of the small GTP-binding protein Rho by cell adhesion and the cytoskeleton. Embo J 18:578-85

Richards RJ, Masek LC, Brown RF (1991) Biochemical and cellular mechanisms of pulmonary fibrosis. Toxicol Pathol 19:526-39

Sane DC, Kontos JL, Greenberg CS (2007) Roles of transglutaminases in cardiac and vascular diseases. Front Biosci 12:2530-45

Sane DC, Moser TL, Parker CJ, Seiffert D, Loskutoff DJ, Greenberg CS (1990) Highly sulfated glycosaminoglycans augment the cross-linking of vitronectin by guinea pig liver transglutaminase. Functional studies of the cross-linked vitronectin multimers. J Biol Chem 265:3543-8

Scarpellini A, Germack R, Johnson T, Muamatsu T, Griffin M, Verderio E (2008) Direct association of transglutaminase-2 with syndecan-4 in the formation of RGDindependent focal adhesions on fibronectin. Biochem Soc Trans. 36:P022.

Scarpellini A, Germack R, Lortat-Jacob H, Muramatsu T, E.A.M. V (2007) Tissue transglutaminase is a heparan sulfate binding protein which cooperates with syndecan- 4 in the formation of focal adhesions on fibronectin. Glycoconjugate $\mathrm{J}$. 24:092

Seeberger PH, Werz DB (2007) Synthesis and medical applications of oligosaccharides. Nature 446:1046-51 
Signorini M, Bortolotti F, Poltronieri L, Bergamini CM (1988) Human erythrocyte transglutaminase: purification and preliminary characterisation. Biol Chem Hoppe Seyler 369:275-81

Skill NJ, Johnson TS, Coutts IG, Saint RE, Fisher M, Huang L, El Nahas AM, Collighan RJ, Griffin M (2004) Inhibition of transglutaminase activity reduces extracellular matrix accumulation induced by high glucose levels in proximal tubular epithelial cells. J Biol Chem 279:47754-62

Small K, Feng JF, Lorenz J, Donnelly ET, Yu A, Im MJ, Dorn GW, 2nd, Liggett SB (1999) Cardiac specific overexpression of transglutaminase II $(\mathrm{G}(\mathrm{h})$ ) results in a unique hypertrophy phenotype independent of phospholipase $\mathrm{C}$ activation. $\mathrm{J}$ Biol Chem 274:21291-6.

Stephens P, Grenard P, Aeschlimann P, Langley M, Blain E, Errington R, Kipling D, Thomas D, Aeschlimann D (2004) Crosslinking and G-protein functions of transglutaminase 2 contribute differentially to fibroblast wound healing responses. J Cell Sci 117:3389-403

Stepp MA, Gibson HE, Gala PH, Iglesia DD, Pajoohesh-Ganji A, Pal-Ghosh S, Brown M, Aquino C, Schwartz AM, Goldberger O, Hinkes MT, Bernfield M (2002) Defects in keratinocyte activation during wound healing in the syndecan-1-deficient mouse. J Cell Sci 115:4517-31

Telci D, Wang Z, Li X, Verderio E, Humphries MJ, Basaga H, Griffin M (2007) TG2-FN complex mediates RGD-independent cell adhesion via interacting with beta1 integrins and their cooperative receptor syndecan-4. Proceedings of the 9th International Conference on transglutaminases and protein cross -linking, Marrakech Morocco:5b

Verderio E, Coombes A, Jones RA, Li X, Heath D, Downes S, Griffin M (2001) Role of the cross-linking enzyme tissue transglutaminase in the biological recognition of synthetic biodegradable polymers. J Biomed Mater Res 54:294-304.

Verderio E, Scarpellini A, Li X, Telci D, Muramatsu T, Melino G, Jones R, Griffin M (2008) Targeted deletion of tgm-2 uncovers a role for extracellular transglutaminase-2 in RhoA downregulation during fibroblast adhesion to fibronectin Biochem Soc Trans 36:P055

Verderio EA, Johnson TS, Griffin M. (2005) Transglutaminases in wound healing and inflammation. In:Bertino JR (ed) Transglutaminases: the family of enzymes with diverse functions. Karger Group Basel

Verderio EA, Telci D, Okoye A, Melino G, Griffin M (2003) A novel RGDindependent cel adhesion pathway mediated by fibronectin-bound tissue transglutaminase rescues cells from anoikis. J Biol Chem 278:42604-14

Wilcox-Adelman SA, Denhez F, Goetinck PF (2002) Syndecan-4 modulates focal adhesion kinase phosphorylation. J Biol Chem 277:32970-7 
Yung S, Woods A, Chan TM, Davies M, Williams JD, Couchman JR (2001) Syndecan-4 up-regulation in proliferative renal disease is related to microfilament organization. Faseb J 15:1631-3

Zehe C, Engling A, Wegehingel S, Schafer T, Nickel W (2006) Cell-surface heparan sulfate proteoglycans are essential components of the unconventional export machinery of FGF-2. Proc Natl Acad Sci U S A 103:15479-84 


\section{Figure Legends}

\section{Figure 1.}

Multiple sequence alignment Allignment (ClustalW) of rat, mouse human and guinea pig liver Transglutaminase 2, showing a conserved putative heparin binding site based on the motif XBBXB (Cardin and Weintraub 1989), where B is either Arg (R) or Lys $(\mathrm{K})$ and $\mathrm{X}$ an hydropathic amino acid.

\section{Figure 2.}

Hypothesis of Transglutaminase-2 (TG2) and HSPG (S4) interactions in the extracellular environment. The long and flexible HS chain with high TG2 affinity suggest HSPGs could bind either free TG2 in the extracellular space or assist in the cells own export of TG2 (1). Alternatively HSPGs could act across cells by binding and localising TG2 on adjacent cells and "sequestering" TG2 from TG2 rich cells thus increasing the local concentration and biological activity of TG2 (2). For example in renal scarring infiltrating fibroblasts expressing S4 could obtain TG2 from tubular epithelial cells. Cell surface bound TG2 could then have a role in both ECM deposition and stabilisation by intra-molecular crosslinking (Fisher et al. 2005; Skill et al. 2004) (3). Cell surface TG2 could also interact with both integrin and cellsurface HSPG (S4) and influence their spatial distribution, synergistic signalling as well as cell adhesion (4). 


\section{Fig 1}

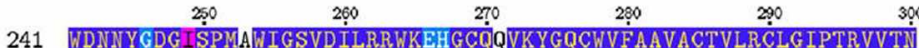
241 WDNNY GDG ISPMAWI GSVDILRRWKEHGCQQVK Y GQCWVFAAVACTVLRCLG IPTRVVTN 241 WDNNY GD GV SPMSWI GSVD ILRRWKNHGCQRVK Y GQCWVFAA VACTVLRCLG IPTRVVTN 241 WDNNYSDGVSPMSUIGSVDILRR WKDYGCQRVKYGQCWVFAAVACTVLRCLG IPTRVVTN Rat TGM2 Mouse TGM2 Human TGM2 Guinea pig TGM2

\section{$\mathrm{XBBXB}$}

X non conserved

I similar

X conserved

区 all match 
Fig 2

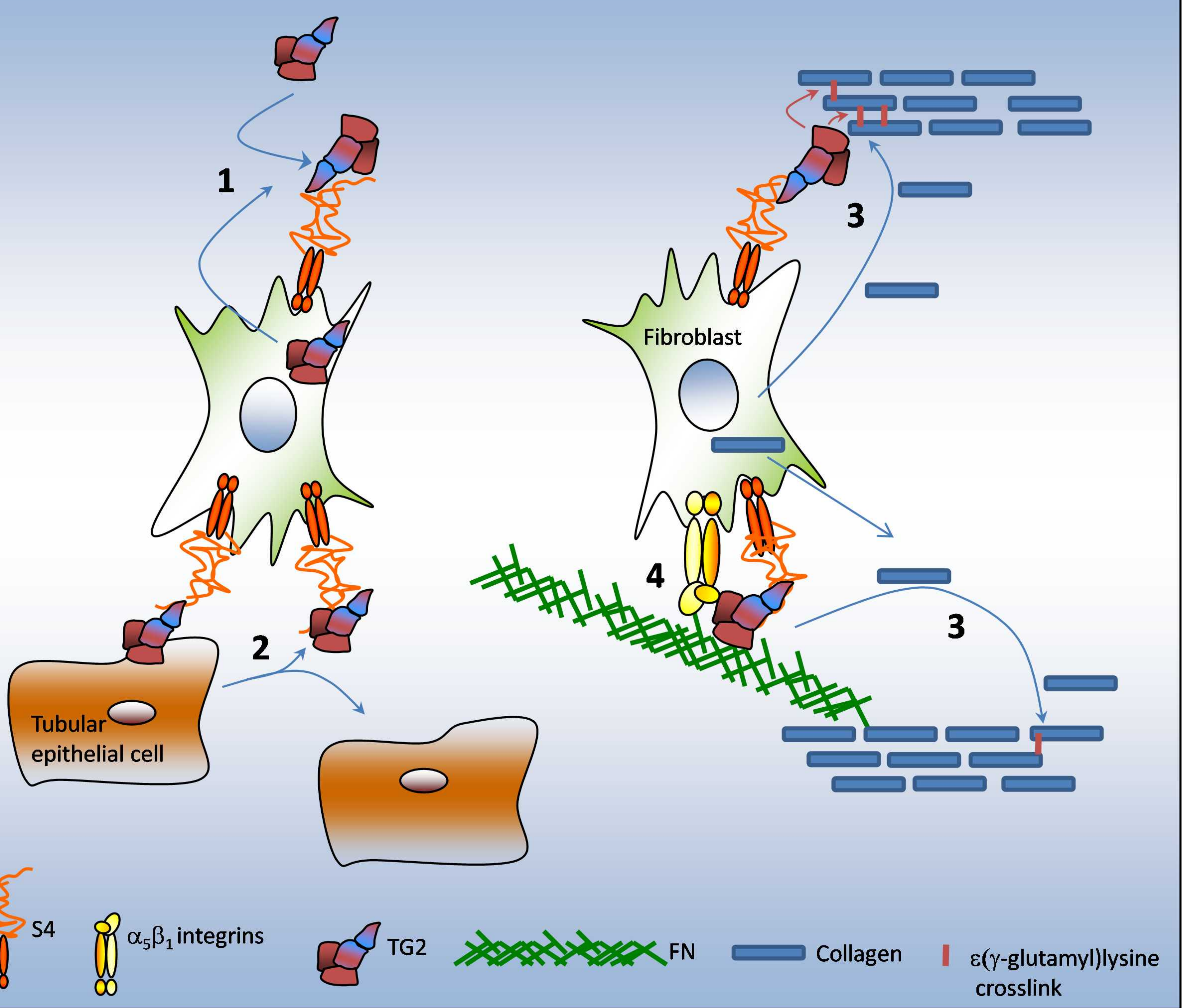

